\title{
Measurement of vegetation parameters and error analysis based on Monte Carlo method
}

\author{
LIANG Boyi ${ }^{1}$, "LIU Suhong ${ }^{2}$ \\ 1. College of Urban and Environment Sciences, Peking University, Beijing 100871, China; \\ 2. Faculty of Geography, Beijing Normal University, Beijing 100875, China
}

\begin{abstract}
In this paper we bring up a Monte Carlo theory based method to measure the ground vegetation parameters, and make quantitative description of the error. The leaf area index is used as the example in the study. Its mean and variance stability at different scales or in different time is verified using both the computer simulation and the statistics of remotely sensed images. And the error of Monte Carlo sampling method is analyzed based on the normal distribution theory and the central-limit theorem. The results show that the variance of leaf area index in the same area is stable at certain scales or in the same time of different years. The difference between experimental results and theoretical ones is small. The significance of this study is to establish a measurement procedure of ground vegetation parameters with an error control system.
\end{abstract}

Keywords: remote sensing; vegetation parameter; error analysis; GLASS LAI

\section{Introduction}

Being one of the main components of the terrestrial biosphere, vegetation accounts for about $50 \%$ of the earth's surface area, and it plays an important role in the earth system by making contribution for atmospheric controlling, water purification, climate regulation, soil and water conservation and so on (Sato et al., 1989; Lean and Rowntree, 1993; Weiss and Baret, 1999; Raich and Tufekciogul, 2000; Ceccato et al., 2001). The vegetation parameters, such as leaf area index (LAI), fractional vegetation coverage (FVC), are key agronomic and ecological parameters, which are of great importance to the study of hydrology, ecology and global change (Fitzgerald et al., 2005; Liang et al., 2015; Barton and North, 2001; Colombo et al., 2003).

The measurement method of vegetation parameters mainly includes ground-based measurement and remote sensing inversion (Ding et al., 2007; Liu et al., 2014; Phillips et al., 1998; González et al., 2008). Since the 1990s, the use of remote sensing data for long time series and large-scale vegetation parameters inversion has become more and more common (Gao 1996; Singh, 1989; Lefsky et al., 2002; Yu et al., 2006; Valentini et al., 2000; Xu et al.,

Received: 2017-06-30 Accepted: 2017-12-08

Foundation: National Natural Science Foundation of China, No.41171262

Author: Liang Boyi, E-mail: liangboyi@pku.edu.cn

"Corresponding author: Liu Shihong, E-mail: liush@bnu.edu.cn 
2013). However, due to the limitations of remote sensing technology, there is a lot of uncertainty in this method (Richey et al., 2002; Benz et al., 2004). Therefore, it is necessary to carry out the ground verification work while retrieving vegetation parameters by using remote sensing data in order to improve the reliability of the results (Chen and Cihlar, 1995; Tan, 2016; Shao et al., 2017). Taking LAI as an example, the ground measurement method mainly includes direct measurement method, digital photography method or using other instruments. Instruments which are frequently used include LAI-2000, Sunfleck Ceptometer, TRAC (Bréda, 2003). Under normal circumstances, people often use system sampling method to select some parts of the study region as the samples, and take the average value of samples as the result of the whole study area, which may result in some error. For the assessment of error, people would normally use their own study area for statistical analysis with no identical standard. So there is still a lack of universal system of error assessment (Manies and Mladenoff, 2000; Mehner et al., 2004).

Monte Carlo method is also called statistical simulation method. Unlike the general deterministic model, the errors need to be analyzed by probabilistic methods (Evensen, 1994; Hastings, 1970; Koehler et al., 2009). Ground-based measurement of vegetation parameters mentioned above is one kind of Monte Carlo random sampling method. In this paper, the variance stability of LAI at different scales and in different time periods was verified using both the computer simulation (micro perspective) and the remote sensing data (macro perspective). The experiment first calculated the theoretical error distribution, and then verified the consistency between real error and theoretical one. By selecting different sampling quantities to measure the vegetation parameters under different vegetation conditions and precision requirements in the study area, we solved the problem of error analysis. Besides, this method was suitable for any scales.

\section{Data and methods}

The vegetation scenarios with three different LAI levels were simulated by computer program. The mean and variance of LAI in each scene were calculated and the frequency curve was generated. The variance stability was observed at different scales. GLASS LAI data was used for verification of variance stability index in different time. In the study, we selected the products for 10 years from 2004 to 2013, taking the 201st day of the year (DOY) as an example, and choosing the three research areas according to their LAI levels.

\subsection{Computer simulation}

Based on fractal tree theory (Frontier, 1987; Lin and Sarabandi, 1999; Weibel, 1999), three scenarios with different vegetation levels (high, medium, low) were randomly generated by computer program. The tree height obeys normal distribution while the position of trees obeys Poisson distribution.

Here, we defined the concept of two-dimensional LAI: first make the side projection of a three-dimensional vegetation scene to the two-dimensional plane, and the sum length of leaves in unit horizontal length was described as two-dimensional LAI. We selected different length as sampling scales $(1,2,4,8,16,32,64$ and 128, unit: pixel, defined as $\Delta \mathrm{x})$ and calculated the mean, variance and frequency curves of the LAI at different scales under the three scenarios (Figure 1). 


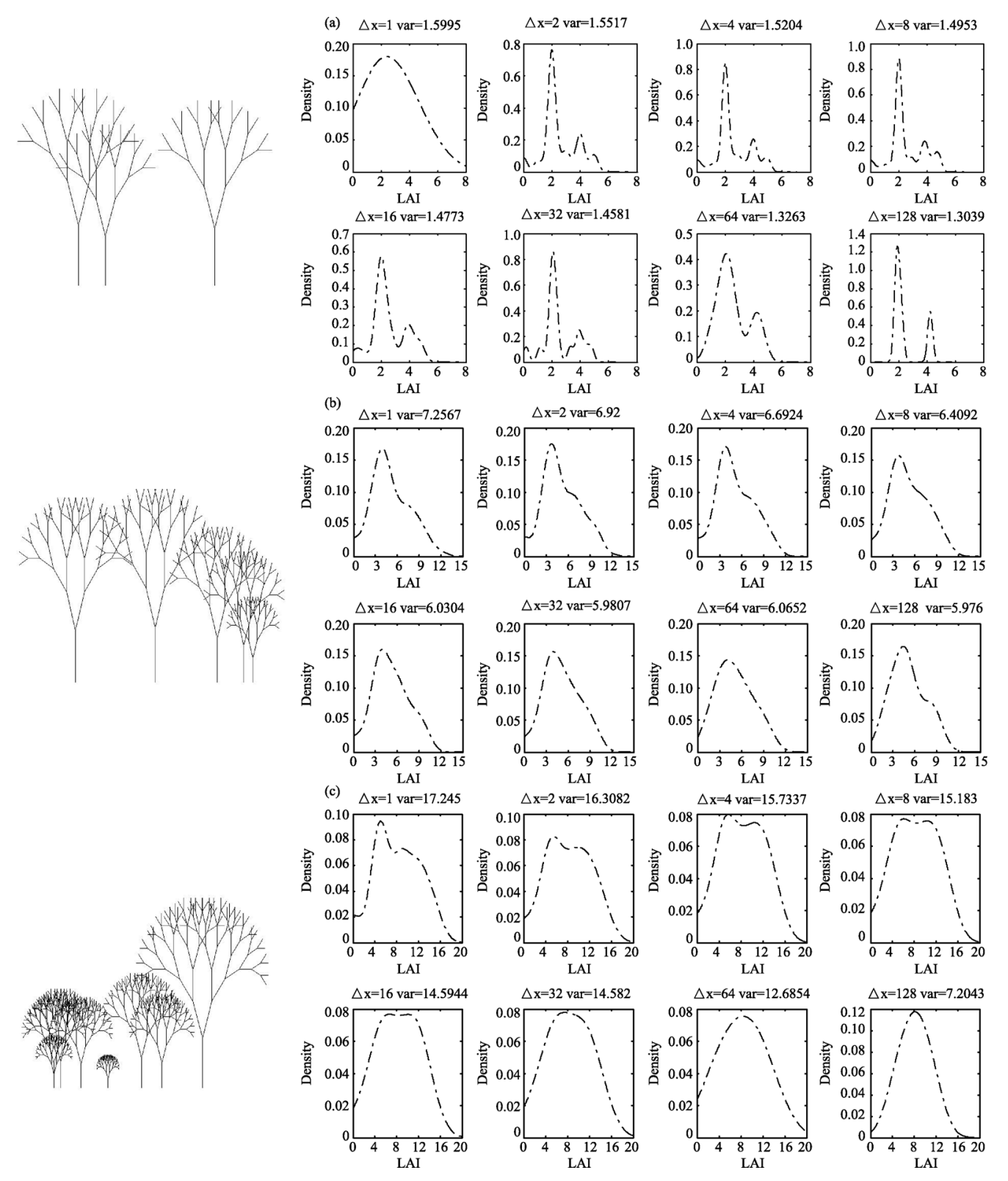

Figure 1 Vegetation scenarios simulated by computer and their leaf area index frequency curves (a. low level; b. median level; c. high level)

In low level scenario, frequency curve of LAI at different scales showed a high consistency except for the circumstances when $\Delta \mathrm{x}$ was 1,128 or 256 . This was because the horizontal length of the whole scene was 512 pixels, so when $\Delta \mathrm{x}$ was equal to 64 or 128 , the number of samples was too small, which was 8 or 4 respectively, resulting in the relative abnormalities of the curve. For the median level scenario, the frequency curves at different scales were more similar to those in low level scene. All curves increased at first and then went down from their peaks. It was not difficult to find that in the computer simulation cases, the frequency curves were irregular regardless of the level of LAI, which did not belong to any kind of common distribution. 
Figure 2 showed the variance scatter plot at different scales in computer simulation cases (low level, median level and high level from the bottom to the top). The variance of LAI decreased with the increase of sampling scale. When the sampling scale was $2,4,8,16,32$ (pixels), the curve was relatively smooth. When the sampling scale rose to 64 and 128 (pixels), the variance began to degrade significantly, especially for the low level and high level cases.

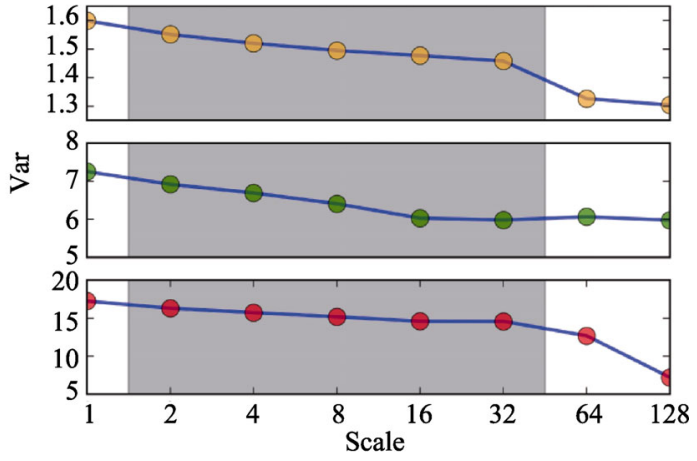

Figure 2 LAI variations in computer simulation

\subsection{Remote sensing data}

Similar to the cases in the computer simulation, three areas (low, median, high level of LAI) were selected in GLASS LAI images (Figure 3, produced by Arcgis 10.0). The three regions were located in Amazon rainforest $\left(55.075^{\circ} \mathrm{W}-70.25^{\circ} \mathrm{W}, 14.925^{\circ} \mathrm{S}-0.025^{\circ} \mathrm{N}\right)$, boreal forest in North Asia $\left(94.975^{\circ} \mathrm{E}-109.925^{\circ} \mathrm{E}, 60.075^{\circ} \mathrm{N}-75.025^{\circ} \mathrm{N}\right)$ and Northwest America $\left(95.075^{\circ} \mathrm{W}-110.025^{\circ} \mathrm{W}, 30.075^{\circ} \mathrm{N}-45.025^{\circ} \mathrm{N}\right)$ respectively. The mean, variance and frequency curves of LAI in different years were calculated as shown in Figure 4.

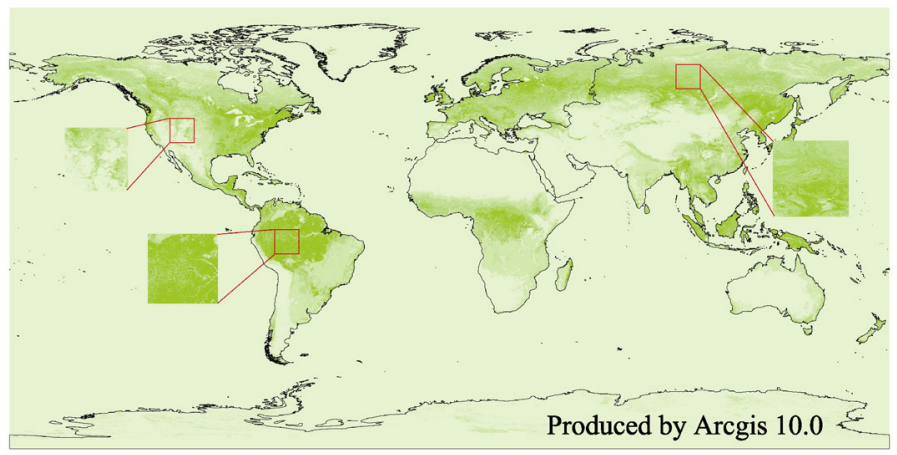

Figure 3 GLASS LAI of the study area

The frequency curves of LAI in the three study regions had different random shapes. The curves have only one "peak" in the high and low level regions, while it was "bimodal" in the median level area. From 2004 to 2013, the variances of LAI in the low, median and high level region ranged from 0.71 to 0.91 , from 1.09 to 1.24 and from 1.46 to 1.84 respectively. In the ten-year time series, variance kept relatively stable.

\subsection{Analysis of error probability}

For a random variable $x$ that obeys normal distribution, the probability density function is (Stein, 1981; Epanechnikov, 1969):

$$
f(x)=\frac{1}{\sqrt{2 \pi} \sigma} e^{-\frac{(x-\mu)^{2}}{2 \sigma^{2}}},-\infty<x<+\infty
$$

where $\mu$ and $\sigma$ are both constant, representing the mean and standard deviation respectively.

And if the variable $x$ obeys normal distribution, its distribution function is: 
(a) year $=2004$ var $=0.90$
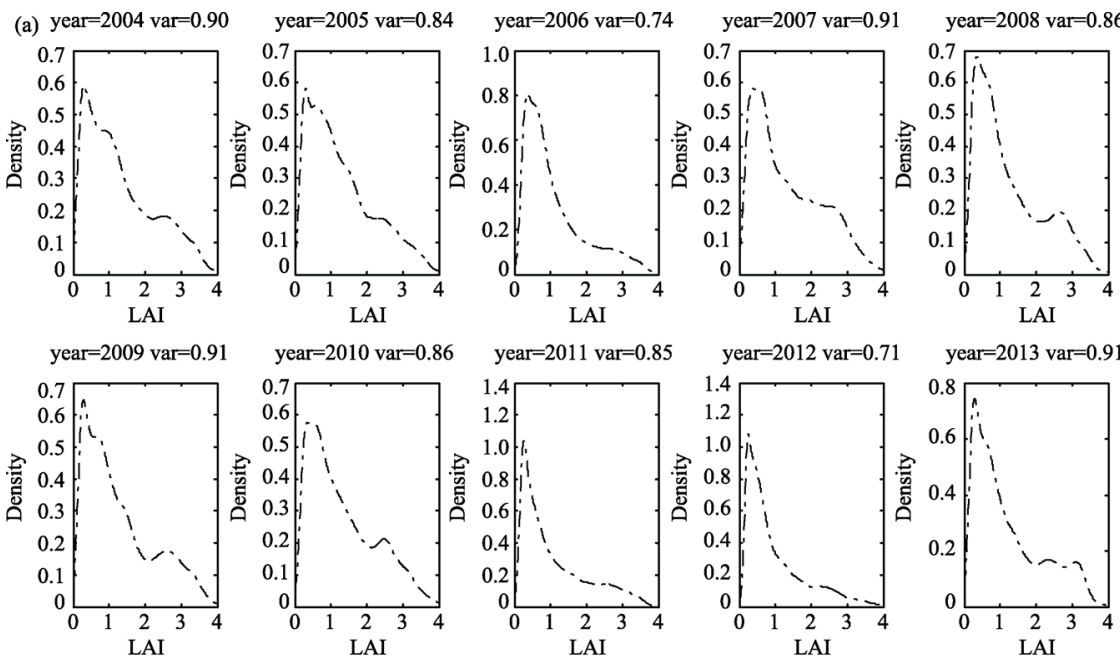

(b) year $=2004$ var $=1.14$
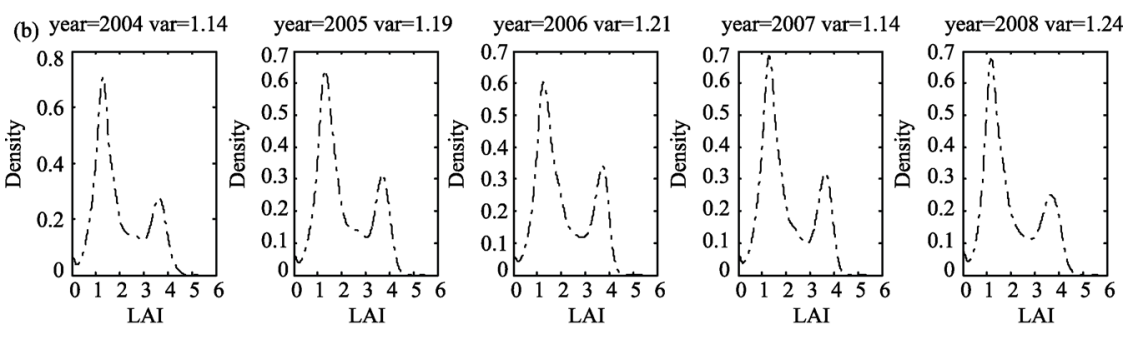

year=2009 var=1.22
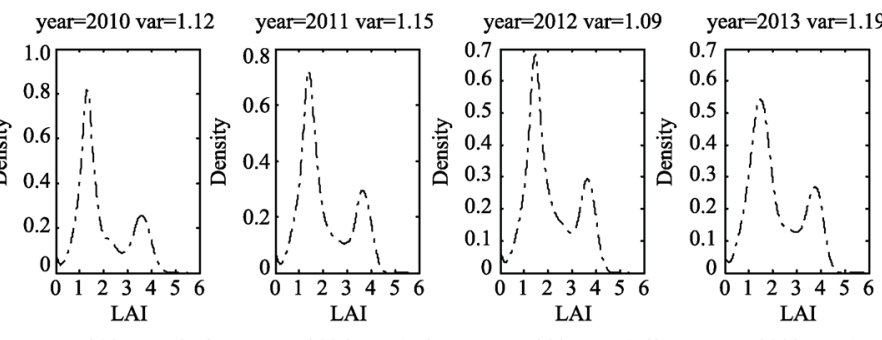

(c) year=2004 var=1.55
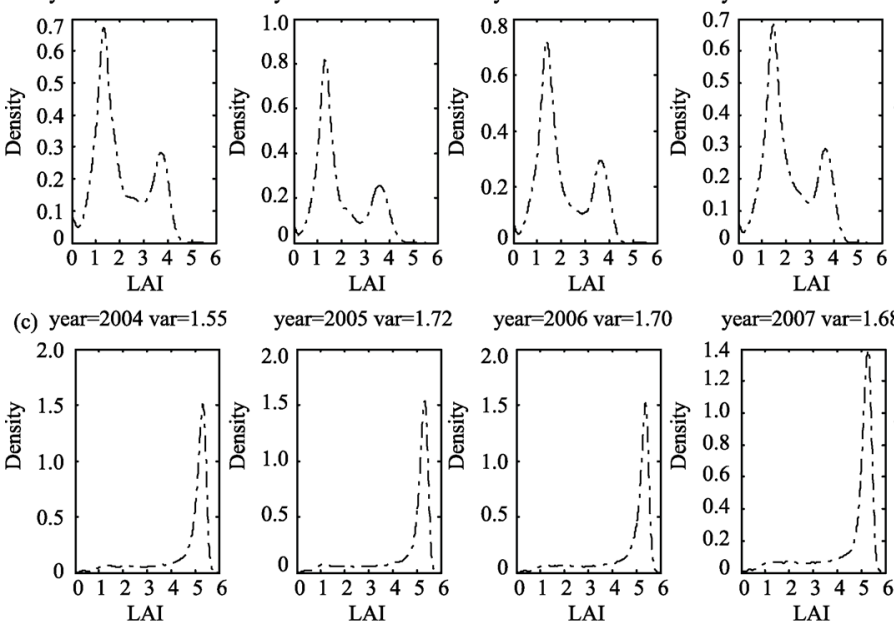

year $=2008$ var $=1.76$
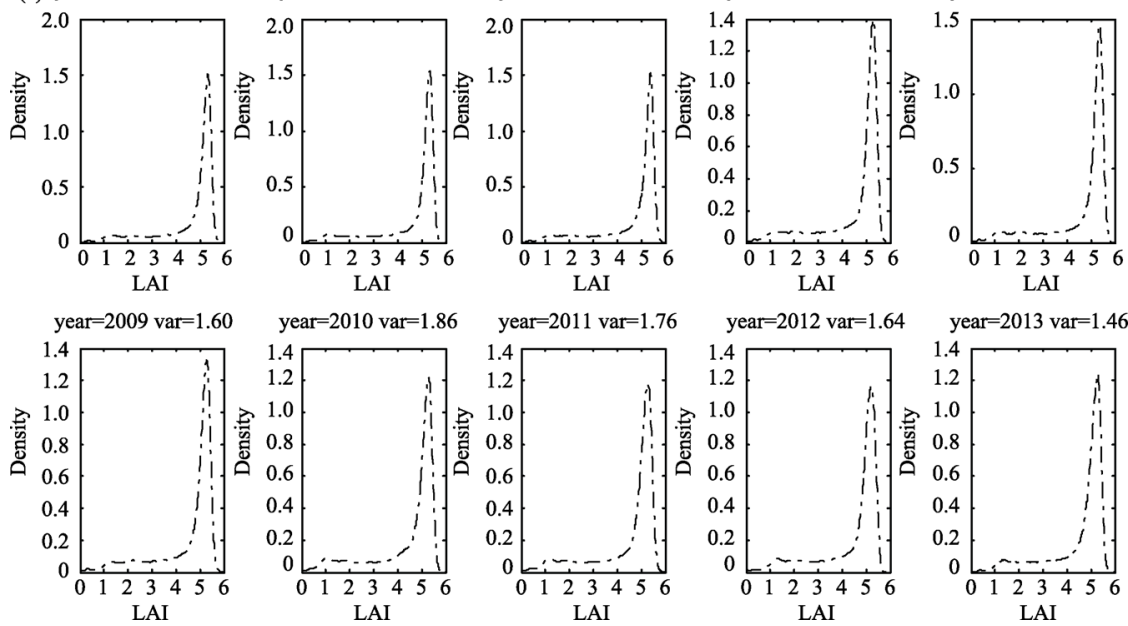

Figure 4 Frequency curves of GLASS LAI (a. low level. b. median level. c. high level) 


$$
F(x)=\frac{1}{\sqrt{2 \pi} \sigma} \int_{-\infty}^{x} e^{-\frac{(x-\mu)^{2}}{2 \sigma^{2}}} d x,-\infty<x<+\infty
$$

And the probability of variable $X$ between $x_{1}$ and $x_{2}$ is:

$$
P\left(x_{1}, x_{2}\right)=F\left(x_{2}\right)-F\left(x_{1}\right)
$$

According to the Lindeberg-Levi theorem (independent distribution center limit theorem) (Ditlevsen et al., 1996; Nishiyama, 2001; Li and Ullha, 1998), if $\xi_{1}, \xi_{2}, \ldots, \xi_{n}, \ldots$ are a series of independent identically distributed random variables, the expect $E \xi_{i}=\mu$, the variance $D \xi_{i}$ $=\sigma^{2}>0, i=1,2, \ldots$, then:

$$
\begin{gathered}
\lim _{n \rightarrow \infty} P\left\{\frac{\sum_{i=1}^{n} \xi_{i}-n \mu}{\sqrt{n} \sigma} \leqslant x\right\}=\frac{1}{\sqrt{2 \pi}} \int_{-\infty}^{x} e^{-\frac{t^{2}}{2}} d t=\Phi_{0}(x), \\
Y_{n}=\frac{\sum_{i=1}^{n} \xi_{i}-n \mu}{\sqrt{n} \sigma} \sim F_{n}(x), \lim _{n \rightarrow \infty} P\left(Y_{n} \leqslant x\right)=\Phi_{0}(x), \lim _{n \rightarrow \infty} F(x)=\Phi_{0}(x), \\
Y_{n}=\frac{\sum_{i=1}^{n} \xi_{i}-n \mu}{\sqrt{n} \sigma} \sim N(0,1), \sum_{i=1}^{n} \xi_{i} \sim N\left(n \mu, n \sigma^{2}\right), \frac{1}{n} \sum_{i=1}^{n} \xi_{i} \sim N\left(\mu, \frac{\sigma^{2}}{n}\right)
\end{gathered}
$$

The theorem states that when we use a sampling method for measuring a variable with an independent identity ( $\mu$ is the mean, $\sigma$ is the standard deviation), the mean value obeys a normal distribution (the mean is $\mu$, the variance is $\sigma^{2} / n$ ) theoretically as long as $n$ is large enough (generally greater than 30), regardless of the distribution of the source data. We applied this theory to the LAI sampling method. Accordingly, when the sampling quantity (number of sampling points or pixels) is large enough, the mean should also obey normal distribution. In addition, the theory also shows that mean value distribution of sampling method is only related to sampling quantity, and has nothing to do with the measurement range. Therefore, we can avoid the scale effect under the hypothesis that the measured results for each time are true values. In this paper, we used the distribution function of normal distribution to calculate theoretical probability of sampling mean value located in different error intervals, establishing the error evaluation system about the sampling method for LAI.

According to the theory described above, a set of ground vegetation parameter measurement processes containing quantitative description of error could be established. We still take LAI as an example. The measurement steps were as follows:

(1) Select the study area on earth. The area could be any size, and we did not need to consider the distribution of vegetation or other geographical parameters in it.

(2) Calculate the frequency histogram of LAI in the entire study area by remote sensing (aerial or aerospace) images, at as many scales as possible. We assumed that the value retrieved by remote sensing images at different scales is true.

(3) Measure LAI in the study area at different scales (from minimum to maximum) or in different time and calculate the variations of them. Through this process, we obtained only one measurement result without variation at the maximum scale (whole area) . The minimum scale referred to the smallest unit which could be achieved technically, such as the highest spatial resolution in remote sensing image. Other scales between the maximum and mini- 
mum ranges were regarded as intermediate scales, and the variance could be stable within certain range. Next, optimal scales were selected artificially according to the requirement of measurement error. Finally, the mean variance at optimal scales or in different periods of time was regarded as the variance of the whole study area.

(4) Use normal distribution theory and the Lindbergh-Levi theorem to calculate the error distribution by using different random sampling quantities. The sampling quantity (which can be sampled at any scale) is determined according to the measurement cost and accuracy requirements. When the error requirement is high, we need more sampling points to make the variance of the mean value smaller and the result closer to the true value of LAI.

(5) Compare the error results deduced from the theory with the real measurement to verify the reliability of error probability distribution.

\section{Result and discussion}

\subsection{Verification of normal distribution}

The six scenarios of computer simulation and GLASS LAI were measured by sampling method. The number of samples was selected as 30, 50, 70, 100, 150, 200, 300 and 500 respectively. For the computer simulation scenario, the sampling unit was the width of one pixel; GLASS LAI was sampled by unit of one pixel. We used computer program to simulate the measurement process of each sampling quantity for 1000 times, and get 1000 sets of mean value. Then we tested the normal distribution of these results shown in Figure 5 with the abscissa being the mean value of LAI under different sampling quantities. In this diagram, the closer the points were to diagonal, the better they obeyed normal distribution. It could be seen that the mean values of all six study areas were close to the normal distribution regardless of sampling quantities. We can find that when the points were centralized in the middle area, the shape was more close to the normal distribution. In contrast, points away from middle had irregular shape of distribution, which was related to the number of simulation cycles.

\subsection{Verification of mean and variation}

For the three vegetation scenarios simulated by computer program, the variance of whole scene was taken as the mean variation at the six median scales, while the variance of the three GLASS LAI regions was taken as the mean value of variation in ten years (from 2004 to 2013). The theoretical value of mean LAI and its variation in three scenarios and three study areas under different sampling quantities were calculated by using the Lindbergh Levi theorem. Then we calculated the real mean LAI and variance for 1000 times by computer simulation, and compared the results with the theoretical ones (Table 1).

The difference between real and theoretical value of variation was small in general. In the computer simulation cases, the maximum error occurred when sampling quantity was 30, and the maximum errors in low, medium and high level scenes were $0.007,0.033$ and 0.037 respectively. As the LAI level increased, the error became smaller gradually. The variance error of GLASS LAI was similar, the maximum error occurred identically when the sampling quantity was 30, and the maximum errors in low, medium and high level scenes were 0.003, 0.001 and 0.009 respectively. The result of the mean was compliant to the theorem, 
that was, as the number of sampling points increased, the sampling mean became more and more convergent to the theoretical value.
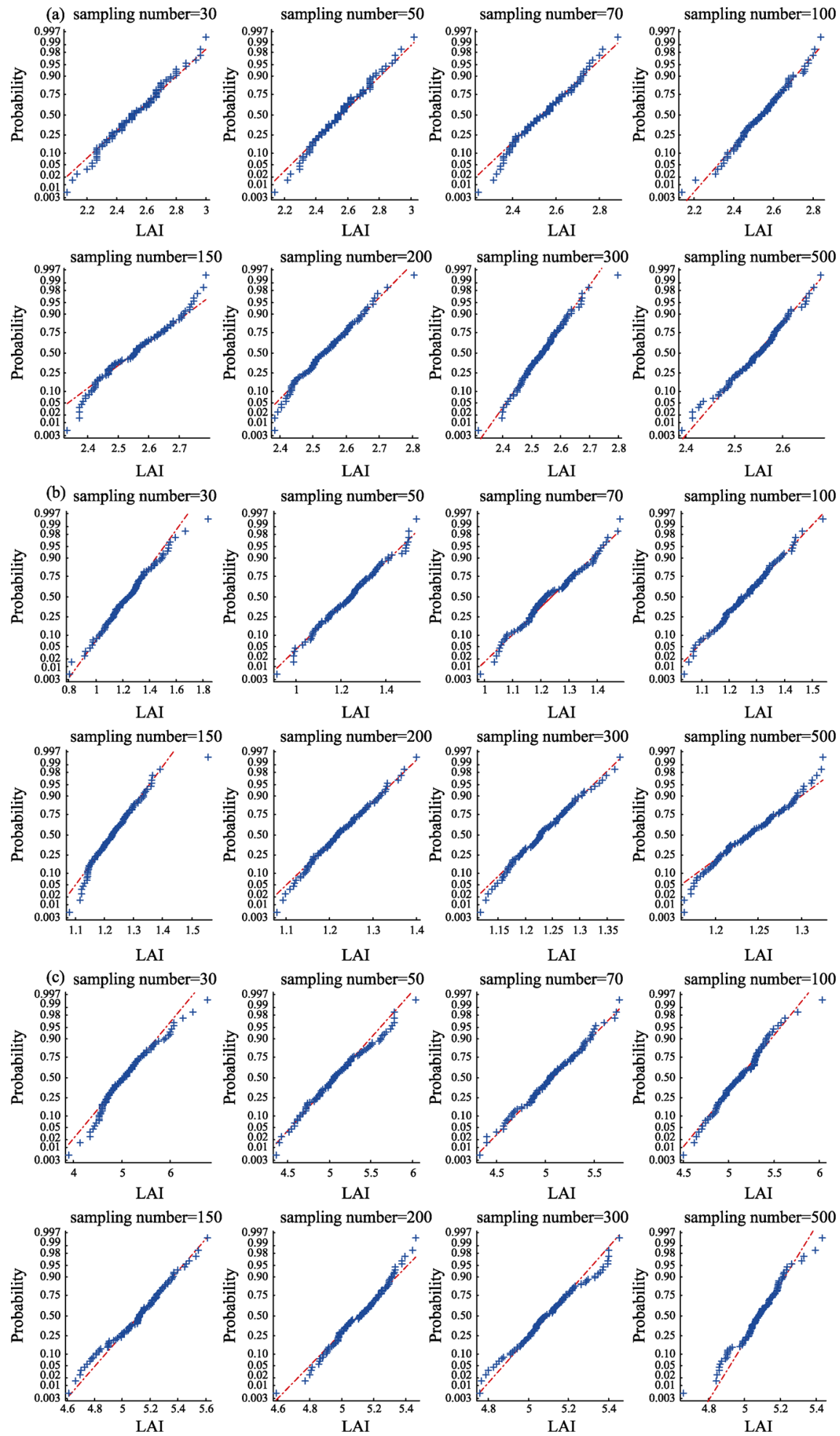

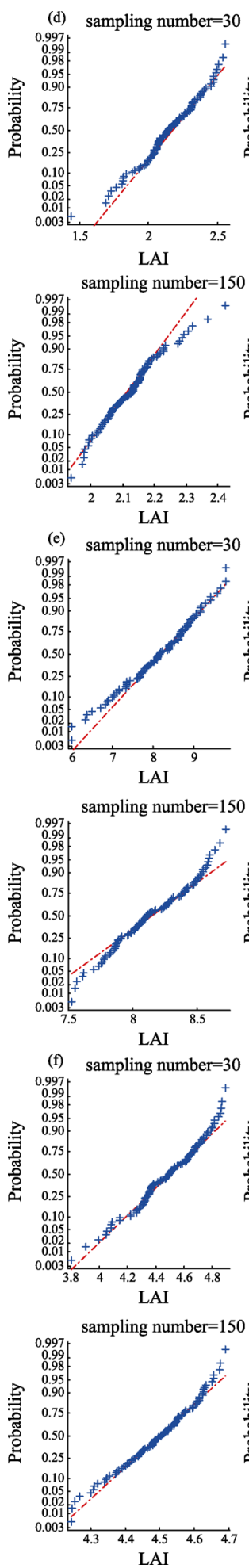
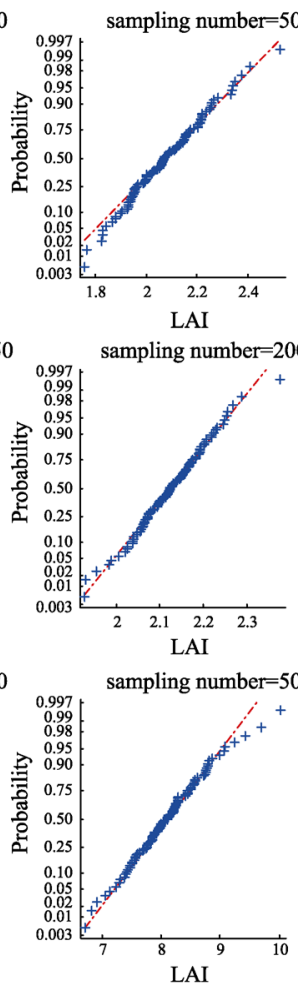

sampling number $=200$
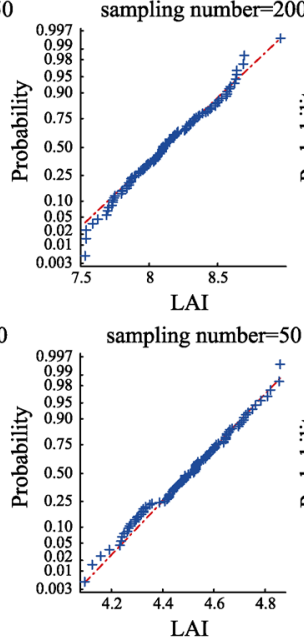

sampling number $=200$

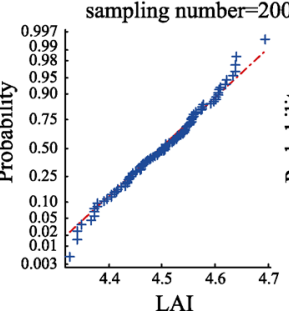

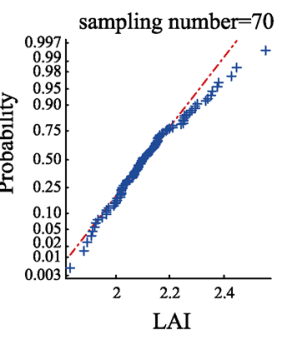
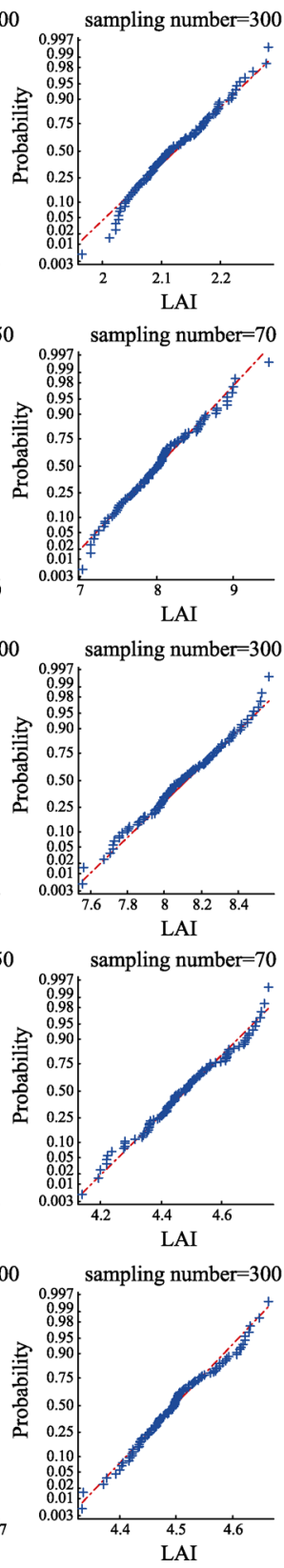
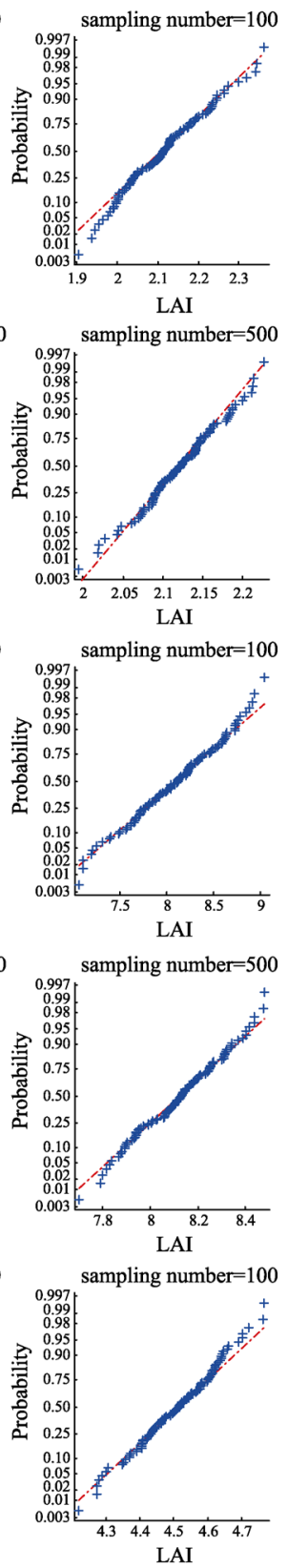

sampling number $=500$

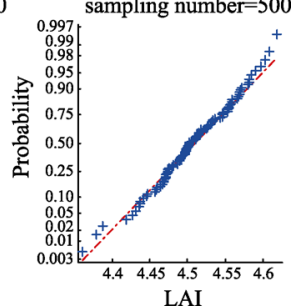

Figure 5 Verification of normal distribution (a. low level of computer simulation; b. low level of GLASS LAI; c. median level of computer simulation; d. median level of GLASS LAI; e. high level of computer simulation; f. high level of GLASS LAI) 
Table 1 Verification of mean and variation

\begin{tabular}{|c|c|c|c|c|c|c|c|c|c|c|c|}
\hline & & & Sam & 30 & 50 & 70 & 100 & 150 & 200 & 300 & 500 \\
\hline \multirow{8}{*}{$\begin{array}{l}\text { Low } \\
\text { level }\end{array}$} & \multirow{4}{*}{$\begin{array}{l}\text { Computer } \\
\text { simulation }\end{array}$} & Variation & Theoretical value & 0.049 & 0.029 & 0.021 & 0.015 & 0.010 & 0.007 & 0.005 & 0.003 \\
\hline & & \multirow{3}{*}{ Mean } & Real value & 0.056 & 0.032 & 0.022 & 0.016 & 0.011 & 0.008 & 0.006 & 0.003 \\
\hline & & & Theoretical value & 2.535 & 2.535 & 2.535 & 2.535 & 2.535 & 2.535 & 2.535 & 2.535 \\
\hline & & & Real value & 2.531 & 2.546 & 2.541 & 2.536 & 2.537 & 2.534 & 2.540 & 2.537 \\
\hline & \multirow{4}{*}{$\begin{array}{c}\text { GLASS } \\
\text { LAI }\end{array}$} & \multirow[t]{2}{*}{ Variation } & Theoretical & 0.028 & 0.017 & 0.012 & 0.008 & 0.006 & 0.004 & 0.003 & 0.002 \\
\hline & & & Real value & 0.031 & 0.018 & 0.013 & 0.009 & 0.006 & 0.005 & 0.003 & 0.002 \\
\hline & & \multirow[t]{2}{*}{ Mean } & Theoretical value & 1.234 & 1.234 & 1.234 & 1.234 & 1.234 & 1.234 & 1.234 & 1.234 \\
\hline & & & Real value & 1.243 & 1.240 & 1.228 & 1.231 & 1.230 & 1.233 & 1.231 & 1.231 \\
\hline \multirow{8}{*}{$\begin{array}{c}\text { Median } \\
\text { level }\end{array}$} & \multirow{4}{*}{$\begin{array}{l}\text { Computer } \\
\text { simulation }\end{array}$} & 11 & Theoretical & 0.212 & 0.127 & 0.091 & 0.064 & 0.042 & 0.032 & 0.021 & 0.013 \\
\hline & & \multirow{3}{*}{ Mean } & Real value & 0.245 & 0.153 & 0.105 & 0.076 & 0.047 & 0.035 & 0.024 & 0.015 \\
\hline & & & Theoretical value & 5.086 & 5.086 & 5.086 & 5.086 & 5.086 & 5.086 & 5.086 & 5.086 \\
\hline & & & Real value & 5.073 & 5.091 & 5.097 & 5.079 & 5.083 & 5.084 & 5.087 & 5.091 \\
\hline & \multirow{4}{*}{$\begin{array}{c}\text { GLASS } \\
\text { LAI }\end{array}$} & on & Theoretical & 0.039 & 0.023 & 0.017 & 0.012 & 0.008 & 0.006 & 0.004 & 0.002 \\
\hline & & \multirow{3}{*}{ Mean } & Real value & 0.038 & 0.024 & 0.016 & 0.011 & 0.008 & 0.006 & 0.004 & 0.002 \\
\hline & & & Theoretical value & 2.115 & 2.115 & 2.115 & 2.115 & 2.115 & 2.115 & 2.115 & 2.115 \\
\hline & & & Real value & 2.099 & 2.122 & 2.118 & 2.117 & 2.113 & 2.115 & 2.115 & 2.115 \\
\hline \multirow{8}{*}{$\begin{array}{l}\text { High } \\
\text { level }\end{array}$} & \multirow{4}{*}{$\begin{array}{l}\text { Computer } \\
\text { simulation }\end{array}$} & Variation & Theoretical value & 0.495 & 0.297 & 0.212 & 0.148 & 0.099 & 0.074 & 0.049 & 0.030 \\
\hline & & \multirow{3}{*}{ Mean } & Real value & 0.532 & 0.352 & 0.270 & 0.176 & 0.122 & 0.085 & 0.054 & 0.035 \\
\hline & & & Theoretical value & 8.086 & 8.086 & 8.086 & 8.086 & 8.086 & 8.086 & 8.086 & 8.086 \\
\hline & & & Real value & 8.089 & 8.096 & 8.087 & 8.081 & 8.103 & 8.090 & 8.081 & 8.096 \\
\hline & \multirow{4}{*}{$\begin{array}{c}\text { GLASS } \\
\text { LAI }\end{array}$} & Variation & Theoretical value & 0.056 & 0.033 & 0.024 & 0.017 & 0.011 & 0.008 & 0.006 & 0.003 \\
\hline & & \multirow{3}{*}{ Mean } & Real value & 0.047 & 0.030 & 0.020 & 0.015 & 0.009 & 0.007 & 0.005 & 0.003 \\
\hline & & & Theoretical value & 4.498 & 4.498 & 4.498 & 4.498 & 4.498 & 4.498 & 4.498 & 4.498 \\
\hline & & & Real value & 4.493 & 4.498 & 4.501 & 4.503 & 4.504 & 4.505 & 4.502 & 4.500 \\
\hline
\end{tabular}

\subsection{Verification of probability error}

In this section, we took high-level area simulated by the computer program as an example. First, we calculated theoretical error distribution by different sampling quantities, and then conducted the verification of theoretical error.

(a)

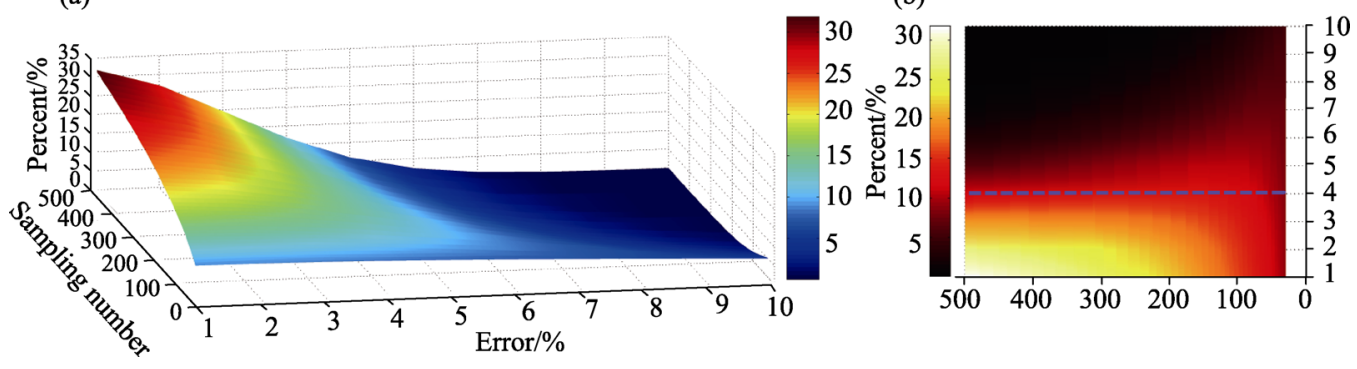

Figure 6 Error distribution (a. three dimensional graph; b. orthographic projection)

Figure 6 showed the quantitative relationship among sampling quantity, ratio of error and mean value (error interval), and frequency of the sampling mean. It could be seen from the 
figure that when the error interval was small, the mean value ratio increased gradually in the error interval with the increase of the sampling quantity. When the error interval was large, the proportion of the mean in the error interval reduced as the sampling quantity increased. Figure $6 \mathrm{~b}$ stood for the vertical projection of Figure 6a above which showed clearly that roughly $4 \%$ error is the dividing line. When the error interval was less than $4 \%$, the sampling mean frequency increased with the increase of sampling quantity and if error interval was greater than $4 \%$, the sampling mean frequency decreased as sampling quantity increased. Namely, with the increase of sampling quantities, the sampling mean distribution was getting closer to the theoretical one, and the overall error was getting smaller.

The error distribution of LAI sampling mean value in high level area under different sampling quantities was simulated by computer program. Each pie chart showed the probability of the mean result error in different intervals by each sampling quantity. The middle region represented the result of the simulation (regarded as the real value), and the peripheral number represented the theoretical value. Table 2 showed the absolute value of the difference between the theoretical and real ratio in different error intervals.

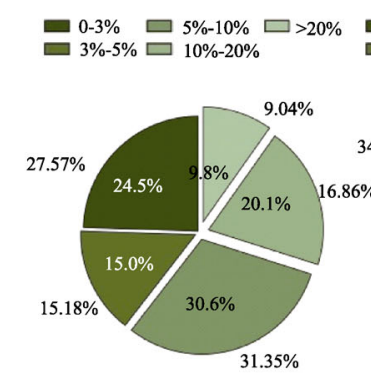

(a)

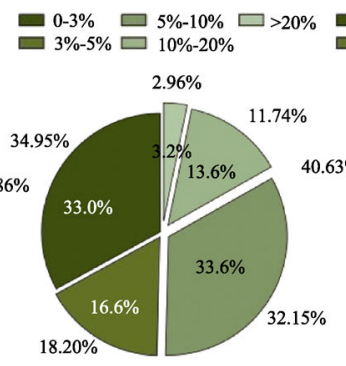

(b)

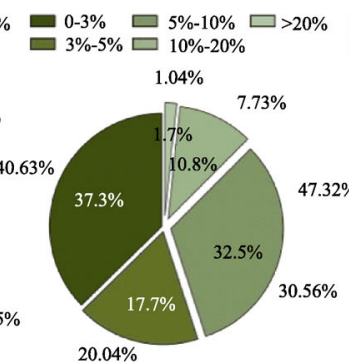

(c)

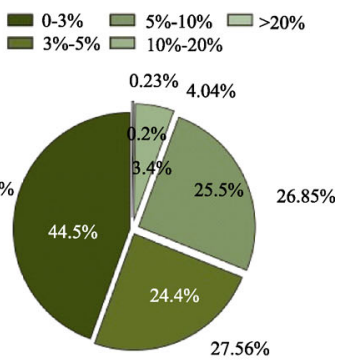

(d)

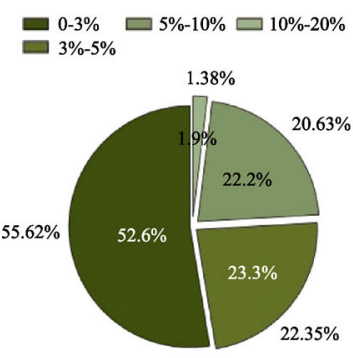

(e)

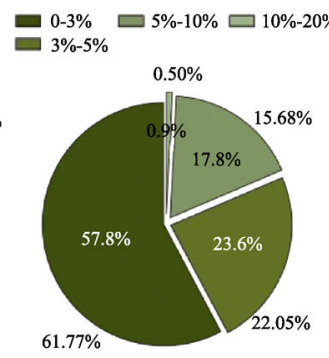

(f)

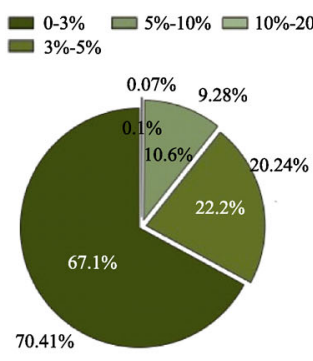

(g)

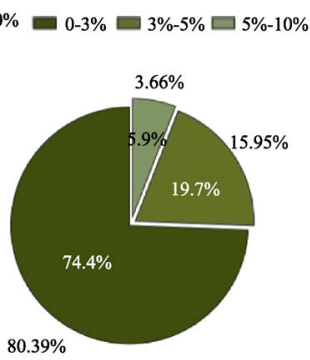

(h)

Figure 7 Error distribution under different sampling quantities (a. 30; b. 50; c. 70; d. 100; e. 150; f. 200; g. 300; h. 500)

Table 2 Statistics of error distribution

\begin{tabular}{ccccccc}
\hline Sampling quantity & $\mathbf{0 - 3 \%}$ & $\mathbf{3 \% - 5 \%}$ & $\mathbf{5 \%}-\mathbf{1 0} \%$ & $\mathbf{1 0} \%-\mathbf{2 0} \%$ & $>\mathbf{2 0} \%$ & Average \\
\hline $\mathbf{3 0}$ & $3.07 \%$ & $0.18 \%$ & $0.75 \%$ & $3.24 \%$ & $0.76 \%$ & $1.60 \%$ \\
$\mathbf{5 0}$ & $1.95 \%$ & $1.60 \%$ & $1.45 \%$ & $1.86 \%$ & $0.24 \%$ & $1.42 \%$ \\
$\mathbf{7 0}$ & $3.33 \%$ & $2.34 \%$ & $1.94 \%$ & $3.07 \%$ & $0.66 \%$ & $2.27 \%$ \\
$\mathbf{1 0 0}$ & $2.82 \%$ & $2.84 \%$ & $1.35 \%$ & $1.36 \%$ & $0.03 \%$ & $1.68 \%$ \\
$\mathbf{1 5 0}$ & $3.20 \%$ & $0.95 \%$ & $1.59 \%$ & $0.52 \%$ & & $1.57 \%$ \\
$\mathbf{2 0 0}$ & $3.97 \%$ & $1.55 \%$ & $2.12 \%$ & $0.30 \%$ & & $1.99 \%$ \\
$\mathbf{3 0 0}$ & $3.31 \%$ & $1.96 \%$ & $1.32 \%$ & $0.03 \%$ & & $1.66 \%$ \\
\hline $\mathbf{5 0 0}$ & $5.99 \%$ & $3.75 \%$ & $2.24 \%$ & & & $3.99 \%$ \\
\hline
\end{tabular}


Table 2 showed that the difference between the real value and theoretical one was quite small. In addition, with the increase of sampling quantity, the proportion of those small error intervals gradually increased, while that of larger error interval gradually decreased. If the sampling quantity was more than 150 , the proportion with error intervals being greater than $20 \%$ was too small to be counted; similarly, if the sampling quantity was more than 500, there were only three kinds of difference in error intervals. Besides, when the sampling quantity was 30 , the largest difference between theoretical and real ratio was $3.24 \%$, being located in the interval of $10 \%-20 \%$; if the sampling quantities were $50,70,150,200,300$, 500 respectively, the largest difference between theoretical and real ratio were $1.95 \%, 3.33 \%$, $3.02 \%, 3.97 \%, 3.31 \%$ and $5.99 \%$, being located in the interval of $0 \%-3 \%$; and when the sampling quantity was 100 , the largest difference between theoretical and real ratio was $2.84 \%$, being located in the interval of $3 \%-5 \%$. This proved the correctness of the previous theoretical error distribution, and showed that using the mean variance at intermediate scales or multiples years as the variance of whole study area had some rationality.

\section{Conclusions}

This paper proposed a vegetation parameter measurement method based on Monte Carlo's theory which included a quantitative error assessment system. The method solves the problem that the error cannot be evaluated when the ground parameters are measured in the past. The complete steps of random sampling measurement of vegetation parameters have high feasibility. This paper has three important conclusions:

(1) When the study area is fixed, the variance of the vegetation parameters at intermediate scales or at the same time of different years has a characteristic of stability, we can take the average value as variance of the whole study area.

(2) According to the normal distribution theory and the Lindbergh-Levi theorem, we can deduce the distribution of the mean of the samples under different sampling quantities, and further calculate the theoretical probability of the mean in different error intervals.

(3) It is found that the error distribution is highly consistent with the theoretical one by simulating the process of measurement, which shows the rationality of error control and the feasibility of sampling method under the error evaluation system. The controlling of the error not only allows the surveyor to have a quantitative expectation for the measurement results, but also makes the allocation of measurement work more rational, in accordance with the requirement of accuracy. We can select the most appropriate sampling quantity of measurement and reduce the cost, as well as improving work efficiency.

\section{Discussion}

The method proposed in this paper is not only applicable to the ground measurement of vegetation parameters, but also practical to other geometric parameters in wide regions. When distributing the layout of the measurement station, the variance characteristics of the geographical parameters in the area are ought to be taken into account. For example, in areas where the parameter differentiation is obvious, the number of measurement should be increased and if the area has high degree of parameter uniformity, we can establish less measurement stations for saving cost. The geographical parameters mentioned include not only 
the ground parameters but also the various variables in the atmosphere which has this kind of stability. This idea of quantitative sampling method has great value for further study in the future.

\section{References}

Barton C V M, North P R J, 2001. Remote sensing of canopy light use efficiency using the photochemical reflectance index: Model and sensitivity analysis. Remote Sensing of Environment, 78: 264-273.

Benz U C, Hofmann P, Willhauck G, 2004. Multi-resolution, object-oriented fuzzy analysis of remote sensing data for GIS-ready information. ISPRS Journal of Photogrammetry and Remote Sensing, 58: 239-258.

Bréda N J J, 2003. Ground-based measurements of leaf area index: A review of methods, instruments and current controversies. Journal of Experimental Botany, 54: 2403-2417.

Ceccato P, Flasse S, Tarantola S, 2001. Detecting vegetation leaf water content using reflectance in the optical domain. Remote Sensing of Environment, 77: 22-33.

Chen J M, Cihlar J, 1995. Plant canopy gap-size analysis theory for improving optical measurements of leaf-area index. Applied Optics, 34: 6211-6222.

Colombo R, Bellingeri D, Fasolini D, 2003. Retrieval of leaf area index in different vegetation types using high resolution satellite data. Remote Sensing of Environment, 86: 120-131.

Ding M J, Zhang Yili, Liu Linshan et al., 2007. The relationship between NDVI and precipitation on the Tibetan Plateau. Journal of Geographical Sciences, 17(3): 259-268.

Ditlevsen O, Mohr G, Hoffmeyer P, 1996. Integration of non-Gaussian fields. Probabilistic Engineering Mechanics, 11: 15-23.

Epanechnikov V A, 1969. Non-parametric estimation of a multivariate probability density. Theory of Probability \& Its Applications, 14: 153-158.

Evensen G, 1994. Sequential data assimilation with a nonlinear quasi-geostrophic model using Monte Carlo methods to forecast error statistics. Journal of Geophysical Research: Oceans, 99: 10143-10162.

Fitzgerald G J, Pinter P J, Hunsaker D J, 2005. Multiple shadow fractions in spectral mixture analysis of a cotton canopy. Remote Sensing of Environment, 97: 526-539.

Frontier S, 1987. Applications of fractal theory to ecology. In: Developments in Numerical Ecology. Berlin Heidelberg: Springer, 335-378.

Gao B C, 1996. NDWI: A normalized difference water index for remote sensing of vegetation liquid water from space. Remote Sensing of Environment, 58: 257-266.

González-Sanpedro M C, Le Toan T, Moreno J et al., 2008. Seasonal variations of leaf area index of agricultural fields retrieved from Landsat data. Remote Sensing of Environment, 112(3): 810-824.

Hastings W K, 1970. Monte Carlo sampling methods using Markov chains and their applications. Biometrika, 57: 97-109.

Koehler E, Brown E, Haneuse S J P, 2009. On the assessment of Monte Carlo error in simulation-based statistical analyses. The American Statistician, 63: 155-162.

Lean J, Rowntree P R, 1993. A GCM simulation of the impact of Amazonian deforestation on climate using an improved canopy representation. Quarterly Journal of the Royal Meteorological Society, 119: 509-530.

Lefsky M A, Cohen W B, Parker G G, 2002. Lidar remote sensing for ecosystem studies: Lidar, an emerging remote sensing technology that directly measures the three-dimensional distribution of plant canopies, can accurately estimate vegetation structural attributes and should be of particular interest to forest, landscape, and global ecologists. BioScience, 52: 19-30.

Li Q, Ullha A, 1998. Estimating partially linear panel data models with one-way error components. Econometric Reviews, 17: 145-166.

Liang B, Liu S, Qu Y et al., 2015. Estimating fractional vegetation cover using the hand-held laser range finder: Method and validation. Remote Sensing Letters, 6: 20-28.

Lin Y C, Sarabandi K, 2014. A Monte Carlo coherent scattering model for forest canopies using fractal-generated 
trees. IEEE Transactions on Geoscience and Remote Sensing, 37: 440-451.

Liu X F, Zhang Jinshui, Zhu Xiufang et al., 2014. Spatiotemporal changes in vegetation coverage and its driving factors in the Three-River Headwaters Region during 2000-2011. Journal of Geographical Sciences, 24(2): 288-302.

Manies K L, Mladenoff D J, 2000. Testing methods to produce landscape-scale presettlement vegetation maps from the US public land survey records. Landscape Ecology, 15: 741-754.

Mehner H, Cutler M, Fairbairn D et al., 2004. Remote sensing of upland vegetation: The potential of high spatial resolution satellite sensors. Global Ecology and Biogeography, 13: 359-369.

Nishiyama Y, 2001. Higher order asymptotic theory for semiparametric averaged derivatives. London School of Economics and Political Science (United Kingdom).

Phillips O L, Malhi Y, Higuchi N et al., 1998. Changes in the carbon balance of tropical forests: Evidence from long-term plots. Science, 282(5388): 439-442.

Raich J W, Tufekciogul A, 2000. Vegetation and soil respiration: Correlations and controls. Biogeochemistry, 48: 71-90.

Richey J E, Melack J M, Aufdenkampe A K et al., 2002. Outgassing from Amazonian rivers and wetlands as a large tropical source of atmospheric $\mathrm{CO}_{2}$. Nature, 416: 617-620.

Sato N, Sellers P J, Randall D A et al., 1989. Effects of implementing the simple biosphere model in a general circulation model. Journal of the Atmospheric Sciences, 46(18): 2757-2782.

Shao Quanqin, Cao Wei, Fan Jiangwen et al., 2017. Effects of an ecological conservation and restoration project in the Three-River Source Region, China. Journal of Geographical Sciences, 27(2): 183-204.

Singh A, 1989. Review article digital change detection techniques using remotely-sensed data. International Journal of Remote Sensing, 10: 989-1003.

Stein C M, 1981. Estimation of the mean of a multivariate normal distribution. The Annals of Statistics, 1135-1151.

Tan Minghong, 2016. Exploring the relationship between vegetation and dust-storm intensity (DSI) in China. Journal of Geographical Sciences, 26(4): 387-396.

Valentini R, Matteucci G, Dolman A J et al., 2000. Respiration as the main determinant of carbon balance in European forests. Nature, 404(6780): 861-865.

Weibel E R, 1999. Fractal geometry: A design principle for living organisms. American Journal of Physiology-Lung Cellular and Molecular Physiology, 261: L361-L369.

Weiss M, Baret F, 1999. Evaluation of canopy biophysical variable retrieval performances from the accumulation of large swath satellite data. Remote Sensing of Environment, 70: 293-306.

Xu L, Myneni R B, Chapin Iii F S et al., 2013. Temperature and vegetation seasonality diminishment over northern lands. Nature Climate Change, 3(6): 581-586.

Yu Q, Gong P, Clinton N et al., 2006. Object-based detailed vegetation classification with airborne high spatial resolution remote sensing imagery. Photogrammetric Engineering \& Remote Sensing, 72(7): 799-811. 